\title{
EL SISTEMA EUROPEO DE TRANSFERENCIA DE CRÉDITOS Y SU IMPLEMENTACIÓN EN UNIVERSIDADES DEL MERCOSUR
}

Guillaume Echevarría* 


\section{RESUMEN}

Este artículo describe el sistema europeo de transferencia de créditos (ECTS), instrumento que permite la movilidad de estudiantes y docentes entre las universidades europeas, por medio del programa de contratos Sócrates-Erasmus, y explora la posibilidad de que este sistema pueda aplicarse a la realidad latinoamericana, particularmente en los países miembros del Mercado Común del Sur, MERCOSUR. Tras representar el modo como opera el ECTS, el artículo concluye con una muestra de la equivalencia de calificaciones y con el examen de las perspectivas de implementación en nuestra región.

\section{ABSTRACT}

This paper describes the European credit transference system (ECTS) that allows students' and faculty's mobility among European universities through the Socrates-Erasmus contract program, and studies the possibility of applying it to the Latinamerican reality, especially to the MERCOSUR members. Besides representing the ECTS' operating methods, the report ends with a sample of the marks' equivalence, and a view of the future of its performance in our region. 


\section{EL SISTEMA EUROPEO DE TRANSFERENCIA DE CRÉDITOS Y SU IMPLEMENTACIÓN EN UNIVERSIDADES DEL MERCOSUR}

El sistema europeo de transferencia de créditos es una herramienta que forma parte de un programa más amplio y completo de movilidad estudiantil, llamado Sócrates-Erasmus, que se implementó en el año 1987 entre los distintos países de la Unión Europea y sus vecinos de Europa central. La política de movilidad estudiantil fue iniciada con el propósito de llegar a una integración europea y seguía a una política de reconocimiento sistemático de diplomas universitarios, ya puesta en marcha anteriormente. El programa Sócrates-Erasmus tenía como objetivo favorecer la movilidad estudiantil y de docentes a través de la implementación de una red de universidades que se fueron enlazando a través de la firma de contratos voluntarios Sócrates-Erasmus. El objetivo de dicho contrato era formalizar enlaces ya existentes o crear nuevas relaciones, y enmarcar la posibilidad de distribuir becas para los intercambios que permitieran cubrir los gastos adicionales de pasaje y estancia en el extranjero. Así, las universidades que firman dicho contrato se comprometen a realizar un determinado nivel de intercambios en las áreas de pregrado, grado, posgrado y doctorado, así como el de docentes para ampliar la oferta de cursos en las universidades de la red.

En el ámbito europeo, el programa Sócrates-Erasmus se ensayó durante los años 1987-1995 con el objetivo de fijar las condiciones óptimas de movilidad estudiantil. A finales de este período se llegó en el año académico 1993-1994 a un total de 100.000 estudiantes que pasaron un mínimo de seis meses en una universidad extranjera. Entonces, se decidió fijar las condiciones definitivas del programa, que sigue funcionando hasta ahora en la Europa de los quince y que se tendrá que adaptar a la escala de la Europa de los veinticinco. Muchos de los países que ingresarán el próximo mes de mayo ya establecieron 
vínculos muy fuertes con los países de la UE a través de la implementación del programa Sócrates-Erasmus, que incluía el mismo sistema de becas que en los países de la UE. En el año 1999-2000 se involucraron en el sistema más de 1.200 universidades del continente europeo. De este modo, fue posible establecer un mismo sistema en países de la UE y exteriores a ella. Esta perspectiva es importante cuando se analiza el proceso iniciado por una red de universidades del MERCOSUR, del cual hablaremos más adelante.

Los beneficios de la movilidad estudiantil a nivel de la Unión Europea son: realizar la integración de las jóvenes generaciones y permitir que los ciudadanos europeos tengan un mejor conocimiento de las distintas culturas de los países que forman la UE. Para el estudiante, es muy positivo (para su curriculum vitae) poder estudiar en un país extranjero sin alargar la duración de sus estudios, con el reconocimiento y la convalidación de las unidades cursadas fuera de su universidad. El beneficio es cultural, lingüístico y puede, también, ser académico (ampliación de la oferta de cursos disponibles en su propia universidad). Para que esta política de movilidad estudiantil se convirtiera en un éxito fue necesario establecer un sistema complejo:

1. En cada país se creó una oficina Sócrates-Erasmus para establecer localmente el programa (política y financieramente).

2. Se implementó un sistema de reconocimiento y contrato mutuo de dos universidades que quisieran intercambiar estudiantes y/o docentes y se centralizaron los datos relativos a cada uno de estos convenios en las oficinas nacionales Sócrates-Erasmus (número de movilidades previstas por universidad y por país).

3. Se inventó un sistema único (European Credit Transfer System ECTS) que permitió traducir en el sistema educativo de cada país el valor de las unidades cursadas en otro así como la escala de calificaciones aplicada para los exámenes.

4. Las universidades con programa de movilidad estudiantil se organizaron de tal modo que, en cada una de ellas, se nombró un responsable que tiene por misión establecer los contratos y el control académico del programa de curso de los estudiantes.

5. Se apoyó esta movilidad estudiantil a través de un sistema de becas distribuidas a todos quienes participaran de los convenios mutuos firmados entre las universidades europeas. 
En estas páginas trataremos de presentar las principales herramientas necesarias para el buen funcionamiento del programa Sócrates-Erasmus y analizar las razones de su éxito en Europa y por qué no se puede ahora imaginar una política europea universitaria sin él. También nos referiremos al esfuerzo de una red de universidades del MERCOSUR para llegar a poner en práctica el mismo sistema y fundar una política regional e internacional de movilidad estudiantil.

\section{El contrato entre universidades}

Es el documento necesario para empezar todo intercambio de estudiantes entre dos universidades europeas. En general, sólo consta de dos páginas, que indican el nombre y la dirección de los responsables del programa en cada una de las dos universidades, así como el de los directores, rectores o decanos de cada facultad o universidad involucrada. También el número de estudiantes de cada nivel (pregrado, grado, posgrado y doctorado), el período previsto para cada movilidad y el número de movilidades docentes y su duración. Una vez firmado por las dos partes, es necesario enviarlo cada año a las dos oficinas Sócrates-Erasmus de los dos países. Las becas serán otorgadas a las universidades únicamente si se realizan las movilidades previstas y, además, el nivel de las becas está relacionado con el porcentaje de movilidad anunciada y realizada por la universidad.

El sistema europeo de transferencia de créditos (ECTS)

La razón de este sistema es hacer posible la convalidación de cursos tomados en la universidad de acogida por la universidad de origen. Para esto, es necesario traducir el contenido, el valor del curso y las calificaciones obtenidas por el estudiante. La base de este sistema es reconocer como unidad académica el semestre, modalidad ya utilizada en el sistema educativo anglosajón. A la suma de trabajo necesaria para aprobar un semestre, se le atribuye un valor de 30 créditos ECTS. Este valor es válido para cualquier nivel académico y para cualquier país o sistema educativo vigente en los países involucrados en el programa Sócrates-Erasmus. El valor de un crédito es equivalente a un 1/30 del volumen normal de trabajo de un semestre para un 
estudiante local. No hay equivalencia sistemática entre el volumen horario de un curso y el número de créditos. En teoría, el sistema ECTS reconoce el valor del trabajo realizado fuera de las aulas (trabajo en casa, informes personales, bibliografía, entre otras actividades). De este modo, el mismo volumen horario de dos carreras equivalentes entre dos países puede resultar en un número de créditos diferentes. La razón de esta diferencia se encuentra en el hecho de que no se estudia de la misma manera en todos los países del mundo. Descubrir esta diferencia es uno de los objetivos de la movilidad estudiantil internacional. En la aplicación del sistema ECTS, para describir las carreras y las unidades de curso, no se debe modificar el idioma en que se dictan las clases -aunque se trate de alumnos extranjeros-; tampoco la estructura y contenido de las carreras ni el método de evaluación de las unidades de curso.

\section{El catálogo informativo}

Este documento permite al estudiante familiarizarse con la institución en la que quiere estudiar durante un semestre o un año. Contiene toda la información necesaria relativa al país, sus costumbres, su sistema universitario, sus carreras y el contenido de cada una (utilizando el sistema ECTS). Es necesario difundir el catálogo informativo a todas las universidades o facultades con las que se tienen vínculos. También es necesario tener dos versiones: una de papel -más formal- y una versión electrónica en la página Web de la universidad para que los estudiantes exteriores la puedan consultar sin problemas de acceso. Su estructura es siempre la misma. La comisión europea propone la guía ECTS en la página Web del programa Sócrates-Erasmus ${ }^{1}$ :

\section{El contrato de estudios}

Es el que firman las tres partes involucradas en el intercambio: el estudiante, la universidad de origen y la universidad de acogida. El estudiante detalla las unidades que desea cursar en la universidad de acogida y las universidades se comprometen a darle acceso a éstas y a

1 Ver sitio en Internet: http://europa.eu.int/comm/education/programmes/Socrates/ects es.html 
convalidarle las unidades escogidas por el semestre que hubiera cursado si no se hubiera ido al extranjero. Es necesario que se firme este convenio antes de viajar por razones de seguros y de recepción de la beca, pero sí se puede modificar una vez que el estudiante haya llegado a la universidad de acogida. El formato es muy simple y, en realidad, consta de una sola página en la que aparecen sobre todo el título, el nivel y el valor (ECTS) de las unidades cursadas, así como el nivel de estudios del alumno. En general, las personas que lo firman en nombre de las universidades o facultades son las responsables de las relaciones internacionales.

\section{La escala de calificaciones ECTS}

Quizás esta parte del sistema ECTS sea la más difícil de entender para los extranjeros al sistema. En realidad se trata de traducir en un lenguaje común las notas sacadas por el estudiante en sus exámenes en el país extranjero para que los responsables académicos de su propia universidad sepan exactamente cuál fue su nivel durante su estadía en la universidad de acogida. También es necesario saber cuáles unidades fueron las que aprobó y qué se le puede convalidar para su carrera. Ya dijimos anteriormente que el hecho de participar al programa Sócrates-Erasmus no debe influir sobre el sistema de exámenes y de calificación. Así, cada universidad entrega a la universidad de origen del estudiante un documento que relata las unidades aprobadas, los créditos obtenidos y las notas para cada unidad según dos sistemas: el ECTS y el local.

El sistema de calificaciones ECTS es muy sencillo. Hay siete calificaciones posibles: dos de ellas califican la insuficiencia (FX y F) y las demás cinco calificaciones (de A a E) se refieren al nivel de calificación en caso de aprobación del examen. Es muy importante señalar que la calificación "A" se atribuye al 10\% de los estudiantes que aprueben con las mejores notas del examen y no a todos los estudiantes que lleguen a un determinado nivel de calificación local. O sea, el sistema ECTS se basa, más bien, en una visión estadística de las calificaciones, lo que permite borrar las diferencias que surgen de maneras diferentes de evaluar (dos docentes dentro de un mismo sistema de calificaciones o entre dos países distintos). 
Escala de calificaciones del sistema ECTS.

\begin{tabular}{|c|c|l|}
\hline $\begin{array}{c}\text { Calificaciones } \\
\text { s } \\
\text { ECTS }\end{array}$ & $\begin{array}{c}\text { Porcentaje aproximado } \\
\text { de estudiantes que } \\
\text { reciben calificación }\end{array}$ & \multicolumn{1}{c|}{ Definición } \\
A & 10 & $\begin{array}{l}\text { SOBRESALIENTE - excelente resultado con } \\
\text { escasas insuficiencias. }\end{array}$ \\
\hline B & 25 & $\begin{array}{l}\text { NOTABLE - resultado superior a la media a } \\
\text { pesar de una serie de insuficiencias. }\end{array}$ \\
\hline C & 30 & $\begin{array}{l}\text { BIEN - buen trabajo en líneas generales, a pesar de } \\
\text { una serie de insuficiencias importantes. }\end{array}$ \\
\hline D & 25 & $\begin{array}{l}\text { SATISFACTORIO trabajo aceptable pero con } \\
\text { considerables insuficiencias. }\end{array}$ \\
\hline E & 10 & $\begin{array}{l}\text { SUFICIENTE - el resultado satisface los criterios } \\
\text { mínimos. }\end{array}$ \\
\hline EX & - & $\begin{array}{l}\text { INSUFICIENTE - se considera necesario un trabajo } \\
\text { suplementario para la concesión de créditos. }\end{array}$ \\
\hline F & - & $\begin{array}{l}\text { DEFICIENTE - es preciso un considerable trabajo } \\
\text { suplementario }\end{array}$ \\
\hline
\end{tabular}

Perspectivas de implementación del sistema ECTS en el MERCOSUR: El sistema STC (Sistema de Transferencia de Créditos)

En el año 2002, la Embajada de Francia en Paraguay trató de colaborar con las principales universidades paraguayas para poner en marcha tal sistema, permitiendo así la movilidad de estudiantes paraguayos en universidades francesas y europeas y la venida de estudiantes franceses a Paraguay. En junio de 2002, tres representantes de las dos universidades mayores del país -la Universidad Nacional de Asunción y la Universidad Católica de Asunción- vinieron a Nancy en el Institut National Polytechnique de Lorraine para intercambiar experiencias y realizar el catálogo informativo relativo a las facultades de ingeniería de ambas universidades. A finales de octubre del mismo año, la Embajada de Francia en Asunción organizó un seminario en el que participaron representantes de universidades (principalmente universidades tecnológicas y facultades de ingeniería) de Argentina, Bolivia, Chile, Paraguay y Uruguay. Allí se presentó el sistema europeo así como las experiencias recientes en ese campo de las dos universidades paraguayas. Muchas de las personas presentes decidieron involucrar a su propia institución en un proceso de movilidad 
estudiantil a través de la activación de un sistema de transferencia de créditos a escala de países del MERCOSUR educativo, y compatible con el sistema europeo. Para ello, se designó a una asociación -la Asociación de Universidades de Ingeniería y Tecnológicas del MERCOSUR (AUITMER) - para armar el proyecto de guía "STC", necesario como marco de movilidad estudiantil dentro de la red de universidades que desearan unirse a la AUITMER.

A principios de mayo de 2003 la AUITMER, en su asamblea de Mendoza, aceptó desempeñar el papel de líder en la implementación del sistema "STC" a nivel MERCOSUR y nombró a un pequeño grupo de miembros, también presentes en el seminario de Asunción, para que trabajara sobre el proyecto de redactar una guía "STC" adaptada a las condiciones del MERCOSUR y compatible con el sistema ECTS. A continuación tuvo lugar en Asunción un segundo seminario para avanzar sobre el mismo tema, también organizado por la Embajada de Francia en el Paraguay. Los representantes de los ministerios de educación presentes en la reunión no quisieron comprometerse en este proyecto, y el grupo de universidades decidió avanzar sin apoyo político, esperando que los estados reconozcan el sistema una vez que exista. El hecho de que sea compatible con el sistema europeo sería una ventaja para tal reconocimiento.

Finalmente, las universidades presentes crearon un grupo de personas (las de la red AUITMER más otras de universidades no involucradas en ésta) para redactar concretamente la guía "STC" y proponerla a las universidades voluntarias así como a la AUITMER y a los participantes del próximo seminario que el Consejo Regional de Desarrollo francés piensa organizar en otro país del Cono Sur.

El Institut National Polytechnique de Lorraine, como muchas de las universidades francesas y europeas (y no sólo las universidades españolas), tiene interés en relacionarse con universidades latinoamericanas, para responder a una demanda de nuestros alumnos que quieren estudiar y, quizás, trabajar en países de este continente. En este marco destaca la doble titulación europeo-latinoamericana, que ya existe entre varias universidades de ambos continentes. Así, estudiantes latinoamericanos pueden conseguir un diploma de ingeniero en Francia, reconocido en Europa, y estudiantes franceses 
el diploma equivalente en el país de América Latina en el que estuvieron. Las empresas europeas que se establecen en países del Cono Sur necesitan contar con ingenieros que conozcan estos países mejor que la antigua generación expatriada de Francia, Italia o España. Los estudiantes doble-titulados responden perfectamente a su demanda. También la colaboración en el campo de la investigación (actividad imprescindible para toda universidad europea) con universidades latinoamericanas podría impulsar el desarrollo del potencial ya existente en los países iberoamericanos. Muchos de los docentes de las universidades de América Latina hicieron su doctorado en Europa $y$ es necesario continuar las relaciones ya existentes ya que, generalmente, son la primera etapa de convenios de intercambio de estudiantes o de proyectos de investigación comunes. Así, a más largo plazo, se podrán desarrollar laboratorios de investigación en las universidades del MERCOSUR, lo que les posibilitaría ofrecer carreras de posgrado y sobre todo de doctorado. Esta última etapa les permitiría estar presentes en la escena mundial al mismo nivel que sus homólogas europeas y norteamericanas. 